\title{
Metastatic Colorectal Cancer Treatment and Survival: the Experience of Major Public Hospitals in South Australia Over Three Decades
}

\author{
David Roder ${ }^{1,8 *}$, Christos S Karapetis ${ }^{2}$, David Wattchow ${ }^{3}$, James Moore ${ }^{4}$, Nimit \\ Singhal $^{5}$, Rohit Joshi ${ }^{6}$, Dorothy Keefe ${ }^{7}$, Kellie Fusco ${ }^{1}$, Dianne Buranyi-Trevarton ${ }^{8}$, \\ Greg Sharplin?', Timothy J Price ${ }^{10}$
}

\begin{abstract}
Background: Registry data from four major public hospitals indicate trends over three decades from 1980 to 2010 in treatment and survival from colorectal cancer with distant metastases at diagnosis (TNM stage IV). Materials and Methods: Kaplan-Meier product-limit estimates and Cox proportional hazards models for investigating disease-specific survival and multiple logistic regression analyses for indicating first-round treatment trends. Results: Two-year survivals increased from $10 \%$ for 1980-84 to $35 \%$ for $2005-10$ diagnoses. Corresponding increases in five-year survivals were from 3\% to $16 \%$. Time-to-event risk of colorectal cancer death approximately halved (hazards ratio: $0.48(0.40,0.59)$ after adjusting for demographic factors, tumour differentiation, and primary sub-site. Survivals were not found to differ by place of residence, suggesting reasonable equity in service provision. About $74 \%$ of cases were treated surgically and this proportion increased over time. Proportions having systemic therapy and/or radiotherapy increased from 12\% in 1980-84 to $61 \%$ for 2005-10. Radiotherapy was more common for rectal than colonic cases (39\% vs $7 \%$ in 2005-10). Of the cases diagnosed in 2005-10 when less than 70 years of age, the percentage having radiotherapy and/or systemic therapy was $79 \%$ for colorectal, $74 \%$ for colon and $86 \%$ for rectum $(\& \mathrm{RS})$ ) cancers. Corresponding proportions having: systemic therapies were $75 \%, 71 \%$ and $81 \%$ respectively; radiotherapy were $24 \%, 10 \%$ and $46 \%$ respectively; and surgery were $75 \%, 78 \%$ and $71 \%$ respectively. Based on survey data on uptake of offered therapies, it is likely that of these younger cases, $85 \%$ would have been offered systemic treatment and among rectum ( $\&$ RS) cases, about $63 \%$ would have been offered radiotherapy. Conclusions: Pronounced increases in survivals from metastatic colorectal cancer have occurred, in keeping with improved systemic therapies and surgical interventions. Use of radiotherapy and/or systemic therapy has increased markedly and patterns of change accord with clinical guideline recommendations.
\end{abstract}

Keywords: Colorectal cancer - metastatic - clinical care - survival trends

Asian Pac J Cancer Prev, 16 (14), 5923-5931

\section{Introduction}

The age-standardized incidence rate of colorectal cancer in Australia is more than twice the estimated world average, whereas the colorectal cancer mortality rate evidently exceeds the world average by only about $8 \%$ (Ferlay et al., 2013). Colorectal cancer is the second leading cancer in Australia both in incidence (excluding non-melanoma skin cancers) and mortality (AIHW, 2013; Ferlay et al., 2013), with only prostate cancer having a higher incidence and lung cancer a higher mortality rate (AIHW, 2013; Ferlay et al., 2013).
Age-standardized colorectal cancer incidence has shown little change in Australia over the past 30 years whereas colorectal death rates have approximately halved (AIHW, 2013). These differences reflect gains in case outcomes, with five-year relative survivals increasing from $48 \%$ for $1982-87$ to $66 \%$ in $2006-10$ diagnoses (AIHW, 2012).

Factors responsible for survival increases are not directly measurable nationally due to a lack of data on diagnostic stage and treatment, although it is thought that survival increases would have occurred both from earlier diagnoses and improved treatments (Kronborg et al., 1987;

${ }^{I}$ Centre for Population Health Research, University of South Australia, ${ }^{2}$ Department of Medical Oncology, Flinders Centre for Innovation in Cancer, ${ }^{3}$ Department of Surgery, Flinders Medical Centre, Flinders University, ${ }^{4}$ Colorectal Surgery, Royal Adelaide Hospital, ${ }^{5}$ Medical Oncology, Royal Adelaide Hospital and University of Adelaide, ${ }^{6}$ Medical Oncology, Lyell McEwin Hospital, ${ }^{7}$ Faculty of Health Sciences, University of Adelaide, ${ }^{8}$ South Australian Health and Medical Research Institute, ${ }^{9}$ Cancer Council South Australia, 10Medical Oncology, Queen Elizabeth Hospital Australia*For correspondence: roder@internode.on.net 
USDHHS, 1990; Hardcastle et al., 1996; Kronborg et al., 1996; Australian Health Technology Advisory Committee, 1997; NHMRC, 1999; Mandel et al., 2000; Jorgensen et al., 2002; Scholefield et al., 2002; ACN, 2005; Hewitson et al., 2008; Department of Health and AIHW, 2008; Schmoll et al., 2012; Cole et al., 2013).

Integrated data on stage and treatment are available in a number of clinical databases, including the South Australian Clinical Cancer Registry (SACCR) for four major public hospitals since 1980 (SACR, 1999). These hospitals serve as major referral centres in addition to providing routine care. While not population-based, their data show stage-specific survival and treatment patterns for about $40 \%$ of colorectal cancers diagnosed in South Australia (one of Australia's eight states and territories) (SACR, 1999; Roder et al., 2015).

SACCR covers referral centres encountering more advanced cancers and potentially greater co-morbidity. While a national survey of colorectal cancers in 50-79 year olds indicated that approximately $18 \%$ had distant metastases at diagnoses, the corresponding figure was $29 \%$ for SACCR cases (NCCI, 2000; Roder et al., 2015). Despite differences in stage and potentially co-morbidity, five-year survivals for SACCR colorectal cases are similar to those for all Australian cases (e.g., 63\% for 2005-10 for SACCR compared with $66 \%$ for Australia overall for 2006-10) (Roder et al., 2015). Risk of colorectal cancer death halved between 1980-86 and 2005-10 diagnoses, the hazards ratio being $0.50(0.45,0.56)$ after adjusting for demographic factors, stage and other tumour characteristics (Roder et al., 2015). Reduced hazards ratios applied to all ACPS stages, including stage D cases (TNM stage 4-distant metastases) where the hazards ratio also approximately halved.

The survival gains for stage D was particularly welcome, given the low survivals for these cancers. SEER U.S.A. data showed five-year survivals in the 5-8\% range during 1983-2000 but with an increase to $13 \%$ five-year survival for 2005-10 diagnoses (Ries et al., 1994; Howlader et al., 2015). Similar trends have been reported for SACCR hospitals (Roder et al., 2015). Gains have been attributed to increased emphasis on systemic therapies, initially using 5-fluorouracil and leucovorin, then irinotecan from the late 1990s, and subsequently oxaliplatin in combination with 5-fluorouracil and leucovorin from circa 2002 (Andre et al., 2004; Martenson et al., 2004). Monoclonal antibody therapies are also available now for targeted therapy, including bevacizumab and cetuximab, and a range of combination systemic therapies are also being used (Price et al., 2014).

Apart from greater use of systemic therapies, radiotherapy has become more common for the control of rectal cancer, and surgical excision or alternatively ablation or embolization of discrete metastatic lesions in the lung and liver is now accepted practice, with these interventions combining to increase survival from metastatic disease (Martenson et al., 2004; Dave et al., 2015; Lubowski, 2015).

With this background, SACCR colorectal cancer data for metastatic cases diagnosed in 1980-2010 are used now, both to indicate trends in survivals and patterns of care, and extent of concordance of treatment with guidelines (USDHHS, 1990; NHMRC 1999; ACN, 2005; Schmoll et al., 2012).

\section{Materials and Methods}

SACCR colorectal cancer data for cases diagnosed with metastatic disease were extracted for 1980-2010 diagnoses, with follow-up data on vital status, death dates and causes to December 31st, 2012. Operational procedures have been described previously (SACR, 1999). Ethical approval was obtained from the SA Health Human Research Ethics Committee.

The SACCR operates under authorization of section 64 of the South Australian Health Care Act (2008) primarily to support quality assurance of service delivery (NCCI, 2000). Patient consent is not legally required for use of non-identifiable data for research authorized under section 64.

Data items covered person descriptors, dates of diagnosis, cancer sub-sites (ICD-O-3 coding or corresponding ICD-9 codes for earlier years), histology type (ICD-O-3 or SNOMED II codes for earlier years), histopathology grade, ACPS stage (an extension of original Dukes' staging to cover distant metastases), and date and cause of death (SACR, 1999). Pre-treatment stage was used.

Death data were obtained from the SA populationbased cancer registry which extracted these data from official South Australian death files, and for deaths occurring outside of South Australia, from the National Death Index at the Australian Institute of Health and Welfare. The extent of loss to follow-up of cancer-registry cases has been checked through active tracing and found to be minimal and to have little effect on calculated survivals (Bonett et al., 1988; SACR, 1999).

Postcode of residence was used to indicate socioeconomic status by quartile, using the Index of Relative Socioeconomic Disadvantage (IRSD), geographic remoteness (classified as metropolitan, regional and remote), and Local Health Network and Medicare Local area of residence (ABS, 1998; Jong et al., 2004; Roder et al., 2015). [Note: While Local Health Networks and Medicare Locals were not operational throughout the study period, they provided useful areas for investigating differences in treatment and outcomes].

Disease-specific survival was indicated using KaplanMeier product-limit estimates, with a censoring of live cases on December 31st, 2012 (STATA, 2012). This method was preferred to relative survival because risks of deaths from competing causes could not be assumed to be equivalent to population norms (an underlying assumption for relative survival) due to the referral of high-risk cases to tertiary referral centres covered by the SACCR (SACR, 1999).

Population-based data have shown disease-specific survival to be a good proxy for relative survival in South Australia. For example, a 1977-2003 colorectal cancer study gave relative survivals of $55 \%$ at five years, $51 \%$ at 10 years and $50 \%$ at 15 years post-diagnosis, which were similar to corresponding disease-specific survivals 
of $56 \%, 51 \%$ and $49 \%$ respectively (Armitage \& Berry, 1987; SACR, 2007; STATA, 2012).

Cox proportional hazards regression analyses also were used to indicate differences in disease-specific survival by person and tumour characteristic, both for single predictors and in a multivariable context, using the same follow-up period and censoring rules as for the Kaplan-Meier analyses (Armitage \& Berry, 1987; SACR, 1999; STATA, 2012). Assumptions underlying the multivariable analyses, including proportionality and lack of co-linearity, were tested and found to be met. When competing risk regression was substituted, results were similar to those of the Cox proportional hazards regression (Armitage \& Berry, 1987; STATA, 2012).

Patterns of first round treatment were assessed by person and tumour characteristic using the Pearson chisquare or Mann-Whitney U test, depending on whether characteristics were measured on a binary, multinominal or ordinal scale (Armitage \& Berry, 1987; STATA, 2012). Multiple logistic regression analysis was employed for multivariable analyses (Armitage \& Berry, 1987; STATA, 2012).

Analyses were undertaken to check for confounding, effect modification and clustering by treatment centre, but did not show statistically significant effects, and so the data presented here are from conventional analyses unadjusted for such effects (STATA, 2012).

\section{Results}

\section{Survival}

Bi-variable analyses: Survivals were $45.5 \%$ at one year, $24.4 \%$ at two years, and $9.6 \%$ at 5 years post diagnosis (Table 1). Survivals were: higher for males in bivariable analyses $(\mathrm{p}<0.001)$ and for more recent diagnostic periods $(\mathrm{p}<0.001)$; and lower with older age at diagnosis $(\mathrm{p}<0.001)$ and poorer tumour differentiation $(\mathrm{p}<0.001)$. Compared with proximal sub-sites, survivals were higher for distal colon, rectum and rectum/sigmoid junction (RS) sub-sites $(\mathrm{p}<0.001)$. Statistically significant differences in survival were not found by place of residence classified by IRSD ( $\mathrm{p}=0.252)$, geographic remoteness $(\mathrm{p}=0.174)$, Local Health Network ( $\mathrm{p}=0.199)$ or Medicare Local $(\mathrm{p}=0.061)$.

Between the 1980-84 and 2005-10 diagnostic periods, survival increased from $22.9 \%$ to $57.1 \%$ at one year from diagnosis, $9.5 \%$ to $34.9 \%$ at two years, and $3.2 \%$ to $16.2 \%$ at five years (Tables 1). Increases applied both to cancers of the colon and rectum (\&RS). For the colon, increases in survival between $1980-84$ and $2005-10$ were from $19.9 \%$ to $53.6 \%$ at one year, $6.6 \%$ to $32.7 \%$ at two years,

Table 1. \% Case Survivals from Colorectal Cancer Diagnosed with Distant Metastases: South Australian Major Public Hospitals, 1980-2010 Diagnoses*

\begin{tabular}{|c|c|c|c|c|c|c|c|}
\hline \multirow[b]{2}{*}{ Groups } & \multicolumn{5}{|c|}{ Period from diagnosis (yrs.) } & \multirow{2}{*}{$\begin{array}{l}\text { Hazards ratios** } \\
(95 \% \text { CLs })\end{array}$} & \multirow[t]{2}{*}{ Pvalue** } \\
\hline & 1 & 2 & 5 & 10 & $\overline{20}$ & & \\
\hline All cases $(n=2192)$ & 45.5 & 24.4 & 9.6 & 6.8 & 6.3 & - & - \\
\hline \multicolumn{8}{|l|}{ Age at diag. (yrs.): } \\
\hline$<40(n=47)$ & 67.8 & 37.2 & 14.1 & 11.3 & - & 1.00 & $<0.001$ \\
\hline $40-49(n=156)$ & 55.1 & 25.4 & 8.9 & 7.8 & 7.8 & $1.29(0.91,1.83)$ & \\
\hline $50-59(n=353)$ & 51.4 & 26.0 & 11.4 & 8.1 & 6.3 & $1.30(0.93,1.81)$ & \\
\hline $60-69(n=569)$ & 51.2 & 29.7 & 12.3 & 8.6 & 8.6 & $1.25(0.90,1.72)$ & \\
\hline $70-79(n=677)$ & 42.4 & 24.2 & 8.4 & 5.3 & - & $1.53(1.11,2.10)$ & \\
\hline $80+(n=390)$ & 29.8 & 13.0 & 5.2 & 5.2 & 5.2 & $2.14(1.54,2.97)$ & \\
\hline \multicolumn{8}{|l|}{ Sex: } \\
\hline Male $(n=1222)$ & 48.4 & 27.4 & 11.2 & 8.1 & 7.3 & 1.00 & $<0.001$ \\
\hline Female $(n=970)$ & 41.9 & 20.6 & 7.6 & 5.2 & 5.2 & $1.17(1.07,1.28)$ & \\
\hline \multicolumn{8}{|l|}{ Differentiation: } \\
\hline Well $(\mathrm{n}=56)$ & 60.1 & 33.2 & 12.7 & 12.7 & 12.7 & 1.00 & $<0.001$ \\
\hline Moderate $(n=1187)$ & 55.4 & 30.4 & 12.5 & 8.3 & 7.5 & $1.11(0.83,1.50)$ & \\
\hline Poor/undiff $(n=635)$ & 28.7 & 14.1 & 5.3 & 4.2 & 4.2 & $1.93(1.43,2.62)$ & \\
\hline$(\mathrm{UK})(\mathrm{n}=314)$ & $(39.4)$ & $(20.9)$ & (6.7) & (5.3) & $(-)$ & - & \\
\hline \multicolumn{8}{|l|}{ Sub-site: } \\
\hline Proximal $(n=602)$ & 37.2 & 17.3 & 7.5 & 5.2 & 5.2 & 1.00 & $<0.001$ \\
\hline Transverse $(n=140)$ & 39.4 & 22.0 & 12.9 & 10.7 & 8.9 & $0.89(0.73,1.09)$ & \\
\hline Distal $(n=631)$ & 46.8 & 24.5 & 9.4 & 6.8 & 6.3 & $0.84(0.75,0.95)$ & \\
\hline Other/unspec. $(n=92)$ & 41.0 & 35.0 & 12.1 & - & - & $0.80(0.62,1.01)$ & \\
\hline Rectosigmoid $(n=207)$ & 53.5 & 30.3 & 10.1 & 6.9 & 6.9 & $0.73(0.62,0.87)$ & \\
\hline Rectum $(\mathrm{n}=520)$ & 52.7 & 28.9 & 10.8 & 7.8 & 7.2 & $0.75(0.66,0.85)$ & \\
\hline \multicolumn{8}{|l|}{ Diagnostic period: } \\
\hline $1980-84(n=156)$ & 22.9 & 9.5 & 3.2 & 3.2 & 3.2 & 1.00 & $<0.001$ \\
\hline $1985-89(n=256)$ & 36.1 & 16.7 & 7.2 & 5.8 & 5.8 & $0.74(0.60,0.90)$ & \\
\hline $1990-94(n=246)$ & 34.6 & 14.8 & 5.2 & 4.2 & 3.7 & $0.81(0.66,1.00)$ & \\
\hline $1995-99(n=435)$ & 44.6 & 23.1 & 7.1 & 6.3 & - & $0.64(0.53,0.78)$ & \\
\hline $2000-04(\mathrm{n}=531)$ & 50.3 & 27.0 & 10.3 & 5.4 & - & $0.59(0.49,0.71)$ & \\
\hline $2005-10(n=568)$ & 57.1 & 34.9 & 16.2 & - & - & $0.47(0.39,0.56)$ & \\
\hline
\end{tabular}

* Kaplan-Meier product-limit disease-specific estimates; date of censoring of live cases-Dec 31, 2012

** Derived from unadjusted Cox proportional hazards regression (data in brackets excluded) Note: statistically significant differences not found by: IRSD ( $\mathrm{p}=0.252)$; ARIA access ( $\mathrm{p}=0.174)$; Medicare Local $(\mathrm{p}=0.061) ; \mathrm{LHN}(\mathrm{p}=0.199)$ 


\section{David Roder et al}

and $2.8 \%$ to $16.0 \%$ at five years (Table 2). Corresponding increases in survival for cancers of the rectum (\&RS) were from $30.3 \%$ to $64.6 \%$ at one year, $16.3 \%$ to $39.8 \%$ at two years, and $3.5 \%$ to $16.6 \%$ at five years. Hazards ratios for 2005-10 compared with the 1980-1984 baseline were similar for the colon at $0.46(0.37,0.58)$ and rectum (\&RS) at $0.48(0.34,0.68)$, although different timings of reductions in hazards ratio were suggested (Table 2 ).
Multivariable analyses

Multivariable analysis results (Table 3) confirm that risk of death from colorectal cancer varied with: i) Age at diagnosis-hazards ratios (95\% confidence limits) increasing with age to $1.94(1.39,2.70)$ for 80 years and over, compared with the reference category of under 40 years; ii) Differentiation-hazards ratio of 1.73 (1.27, 2.35) for poorly differentiated and undifferentiated

Table 2. \% Case Survivals from Colorectal Cancer Diagnosed with Distant Metastases, by Diagnostic Period: South Australian Major Public Hospitals, 1980-2010 Diagnoses*

\begin{tabular}{|c|c|c|c|c|c|c|c|c|}
\hline \multirow[b]{2}{*}{ Site } & \multirow[b]{2}{*}{ Diagnostic period } & \multicolumn{5}{|c|}{ Period from diagnosis (yrs.) } & \multirow{2}{*}{$\begin{array}{c}\text { Hazards ratios** } \\
(95 \% \text { CLs })\end{array}$} & \multirow[t]{2}{*}{ Pvalue $* *$} \\
\hline & & 1 & 2 & 5 & 10 & $\overline{20}$ & & \\
\hline \multirow[t]{6}{*}{ Colon } & $1980-84(n=110)$ & 19.9 & 6.6 & 2.8 & 2.8 & 2.8 & 1.00 & \multirow[t]{6}{*}{$<0.001$} \\
\hline & $1985-89(n=166)$ & 31.5 & 16.6 & 8.7 & 6.3 & 6.3 & $0.71(0.56,0.92)$ & \\
\hline & $1990-94(\mathrm{n}=165)$ & 33.7 & 14.7 & 5.7 & 4.2 & - & $0.75(0.59,0.97)$ & \\
\hline & $1995-99(n=293)$ & 43.0 & 21.9 & 6.3 & 5.8 & - & $0.64(0.51,0.80)$ & \\
\hline & $2000-04(n=341)$ & 43.5 & 21.1 & 8.4 & 4.3 & - & $0.64(0.51,0.80)$ & \\
\hline & $2005-10(n=390)$ & 53.6 & 32.7 & 16.0 & - & - & $0.46(0.37,0.58)$ & \\
\hline Rectum & $1980-84(n=46)$ & 30.3 & 16.3 & 3.5 & 3.5 & 3.5 & 1.00 & \multirow[t]{6}{*}{$<0.001$} \\
\hline \multirow[t]{5}{*}{ (\&R/Sig) } & $1985-89(n=90)$ & 44.4 & 16.9 & 4.8 & 4.8 & 4.8 & $0.83(0.57,1.20)$ & \\
\hline & $1990-94(n=81)$ & 36.3 & 14.9 & 4.1 & 4.1 & 2.7 & $0.97(0.67,1.41)$ & \\
\hline & $1995-99(n=142)$ & 48.0 & 25.5 & 8.8 & 7.2 & - & $0.68(0.48,0.96)$ & \\
\hline & $2000-04(n=190)$ & 62.2 & 37.1 & 13.6 & 7.4 & - & $0.55(0.39,0.76)$ & \\
\hline & $2005-10(n=178)$ & 64.6 & 39.8 & 16.6 & - & - & $0.48(0.34,0.68)$ & \\
\hline
\end{tabular}

*Kaplan-Meier product-limit disease-specific estimates; date of censoring of live cases-Dec 31, 2012; **Derived from unadjusted Cox proportional hazards regression

Table 3. Hazards Ratios (95\% CLs) for Death from Colorectal Cancer Diagnosed with Distant Metastases: South Australian Major Public Hospitals, 1980-2010 Diagnoses* 3 Multivariable Analyses

\begin{tabular}{|c|c|c|c|}
\hline & \multicolumn{3}{|c|}{ Hazards ratios } \\
\hline & Colorectal $(\mathrm{n}=2192)$ & Colon $(n=1465)$ & $\operatorname{Rectum}(\& \mathrm{R} / \mathrm{Sig})(\mathrm{n}=727)$ \\
\hline \multicolumn{4}{|l|}{ Age at diagnosis (yrs): } \\
\hline$<40(\mathrm{n}=47)$ & 1.00 & $(n=32) \quad 1.00$ & $(n=15) \quad 1.00$ \\
\hline $40-49(n=156)$ & $1.19(0.84,1.69)$ & $(\mathrm{n}=101) 1.34(0.86,2.07)$ & $(\mathrm{n}=55) \quad 0.83(0.45,1.54)$ \\
\hline $50-59(n=353)$ & $1.21(0.87,1.68)$ & $(\mathrm{n}=230) 1.33(0.88,2.01)$ & $(\mathrm{n}=123) 0.97(0.55,1.73)$ \\
\hline $60-69(n=569)$ & $1.19(0.86,1.65)$ & $(\mathrm{n}=339) 1.32(0.88,1.98)$ & $(\mathrm{n}=230) 0.90(0.51,1.95)$ \\
\hline $70-79(n=677)$ & $1.43(1.04,1.98)$ & $(\mathrm{n}=477) 1.58(1.06,2.36)$ & $(\mathrm{n}=200) 1.11(0.63,1.95)$ \\
\hline $80+(n=390)$ & $1.94(1.39,2.70)$ & $(\mathrm{n}=286) 2.01(1.33,3.03)$ & $(\mathrm{n}=104) 1.78(0.99,3.20)$ \\
\hline \multicolumn{4}{|l|}{ Sex: } \\
\hline Male $(n=1222)$ & 1.00 & $(\mathrm{n}=789) 1.00$ & $(n=433) 1.00$ \\
\hline Female $(n=970)$ & $0.92(0.84,1.01)$ & $(\mathrm{n}=676) 1.01(0.90,1.13)$ & $(\mathrm{n}=294) 0.72(0.61,0.85)$ \\
\hline \multicolumn{4}{|l|}{ Differentiation: } \\
\hline Well $(n=56)$ & 1.00 & $(n=34) \quad 1.00$ & $(n=22) \quad 1.00$ \\
\hline Moderate $(n=1187)$ & $1.08(0.80,1.46)$ & $(\mathrm{n}=768) 1.15(0.78,1.70)$ & $(\mathrm{n}=419) 1.08(0.66,1.76)$ \\
\hline Poor/ undiff. $(n=635)$ & $1.73(1.27,2.35)$ & $(n=448) 1.73(1.16,2.58)$ & $(\mathrm{n}=187) 2.14(1.29,3.54)$ \\
\hline UK $(n=314)$ & $1.68(1.22,2.32)$ & $(\mathrm{n}=215) 1.70(1.12,2.58)$ & $(\mathrm{n}=99) \quad 1.80(1.05,3.07)$ \\
\hline \multicolumn{4}{|l|}{ Sub-site: } \\
\hline Proximal $(n=602)$ & 1.00 & $(\mathrm{n}=602) 1.00$ & - \\
\hline Transverse $(n=140)$ & $0.94(0.77,1.15)$ & $(\mathrm{n}=140) 0.97(0.79,1.20)$ & - \\
\hline Distal $(n=631)$ & $0.92(0.81,1.04)$ & $(\mathrm{n}=631) 0.91(0.80,1.03)$ & - \\
\hline Other/unspec. $(n=92)$ & $0.91(0.71,1.12)$ & $(n=92) \quad 0.91(0.71,1.16)$ & - \\
\hline Rectosigmoid $(\mathrm{n}=207)$ & $0.83(0.70,0.99)$ & - & $(\mathrm{n}=207) 1.00$ \\
\hline Rectum $(n=520)$ & $0.84(0.74,0.96)$ & - & $(\mathrm{n}=520) 0.91(0.76,1.10)$ \\
\hline \multicolumn{4}{|l|}{ Diagnostic period: } \\
\hline $1980-84(n=156)$ & 1.00 & $(\mathrm{n}=110) 1.00$ & $(\mathrm{n}=46) \quad 1.00$ \\
\hline $1985-89(n=256)$ & $0.73(0.59,0.90)$ & $(n=166) 0.73(0.56,0.94)$ & $(\mathrm{n}=90) \quad 0.77(0.53,1.13)$ \\
\hline $1990-94(n=246)$ & $0.85(0.69,1.06)$ & $(\mathrm{n}=165) 0.79(0.64,1.03)$ & $(\mathrm{n}=81) \quad 1.08(0.73,1.59)$ \\
\hline $1995-99(\mathrm{n}=435)$ & $0.69(0.57,0.84)$ & $(\mathrm{n}=293) 0.70(0.55,0.89)$ & $(\mathrm{n}=142) 0.70(0.48,1.00)$ \\
\hline $2000-04(n=531)$ & $0.60(0.49,0.73)$ & $(\mathrm{n}=341) 0.65(0.51,0.82)$ & $(\mathrm{n}=190) 0.53(0.37,0.76)$ \\
\hline $2005-10(n=568)$ & $0.48(0.40,0.59)$ & $(\mathrm{n}=390) 0.48(0.38,0.61)$ & $(\mathrm{n}=178) 0.49(0.34,0.71)$ \\
\hline
\end{tabular}

*Derived from Cox proportional hazards regression, adjusted for non-significant ( $\mathrm{p}>0.05$ ) associations with IRSD quartile; remoteness of residence; Local Health Network; Medicare Local (see text). Date of censoring of live cases-Dec 31, 2012 
Metastatic Colorectal Cancer Treatment and Survival: Experience of Major Public Hospitals in South Australia

cancers compared with the well-differentiated reference category; iii) Sub-site-hazards ratio of $0.83(0.70,0.99)$ for rectum/sigmoid junction and $0.84(0.74,0.96)$ for rectum compared with the proximal colon; $i v$ ) Diagnostic year-hazards ratios decreasing from the 1980-86 reference period to $0.48(0.40,0.59)$ for $2005-10$.

Similar trends occurred by age, differentiation, and diagnostic period for colon cases and those with a rectum (\&RS) primary site, although the age gradient appeared to be less pronounced for rectum ( $\& \mathrm{RS})$ and the reduction in hazards ratio to occur later for rectum ( $\&$ RS) than colon cases (Table 3).

Multi-variable results were similar to those from bivariable analyses except that the higher hazards ratio for females was no longer elevated after adjustment for age and other co-variables (Table 3 ). A lower hazards ratio of $0.72(0.61,0.85)$ applied for female than male rectum (\&RS) cases. As for the unadjusted analyses, hazards ratios did not vary significantly by place of residence $(\mathrm{p}>0.05)$.

\section{Treatment}

\section{Surgery}

Bi-variable analyses: A total of 1,619 cases (73.9\%) had surgical treatment, with the proportion increasing from $67.0 \%$ for $1980-89$ and $75.6 \%$ for $1990-2010$ diagnoses (MWp<0.001), but without evidence of a continuing increase after $1990(\mathrm{MWp}=0.999)$. Corresponding increases were from $65.2 \%$ to $77.1 \%$ for colon cases and more marginally from $70.6 \%$ to $72.2 \%$ for rectum (\&RS) cases.

Multivariable analyses: Multiple logistic regression analysis, with age, sex, differentiation, sub-site and diagnostic period as predictors, indicated that only diagnostic period was predictive of surgical treatment. Compared with 1980-89 diagnoses, the relative odds (95\% confidence limits) of surgery for 1990-2010 were $2.25(1.75,2.89)$ for all colorectal cases, $2.96(2.17,4.04)$ for colon and $1.41(0.91,2.20)$ for rectum $(\& R S)$ cases. A time trend was not evident for successive 5-6 year epochs during 1990-2010 ( $\mathrm{p}>0.10)$.

\section{Radiotherapy/systemic therapies}

Bi-variable analyses: A total of 921 cases $(42.0 \%)$ had systemic therapy and/or radiotherapy, with this proportion increasing from $11.5 \%$ for $1980-84$ to $61.0 \%$ for 2005 $10(\mathrm{MWp}<0.001)$. Corresponding increases were from $11.8 \%$ to $55.4 \%$ for colon and $10.9 \%$ to $73.5 \%$ for rectum (\&RS) cases. Percentages having these therapies were also higher for: $i$ ) Younger ages at diagnosis: e.g., 70.3\% for cases under 50 years, decreasing to $13.1 \%$ for those aged 80 years and over $(\mathrm{MWp}<0.001)$. Corresponding decreases were from $67.7 \%$ to $11.5 \%$ for colon and $75.4 \%$ to $17.3 \%$ for rectum (\&RS) cases; $i$ ) Rectum (\&RS) cases at $51.2 \%$ than colon cases at $37.5 \%(\mathrm{p}<0.001)$; A total of 314 colorectal $(14.3 \%)$ had radiotherapy, increasing from $1.9 \%$ for $1980-1984$ to $16.9 \%$ for $2005-10$ (MWp<0.001).

Table 4. Relative Odds (95\% Confidence Limits) of Adjuvant Therapies for Colorectal Cancers Diagnosed with Distant Metastases: South Australian Major Public Hospitals, 1980-2010 Cases [N=2192]*-3 Multiple Logistic Regression Analyses -

\begin{tabular}{|c|c|c|c|}
\hline Groups & Radiotherapy (n=314) & Systemic $(\mathrm{n}=811)$ & Either $(n=921)$ \\
\hline \multicolumn{4}{|l|}{ Age at diagnosis (yrs.): } \\
\hline$<40(\mathrm{n}=47)$ & 1.00 & 1.00 & 1.00 \\
\hline $40-49(n=156)$ & $1.19(0.47,2.99)$ & $0.87(0.39,1.95)$ & $1.29(0.58,2.88)$ \\
\hline $50-59(n=353)$ & $1.15(0.48,2.76)$ & $0.60(0.28,1.28)$ & $0.82(0.39,1.72)$ \\
\hline $60-69(n=569)$ & $0.98(0.42,2.32)$ & $0.40(0.19,0.84)$ & $0.47(0.23,0.99)$ \\
\hline $70-79(n=677)$ & $0.62(0.26,1.48)$ & $0.17(0.08,0.36)$ & $0.22(0.11,0.47)$ \\
\hline $80+(n=390)$ & $0.24(0.09,0.62)$ & $0.04(0.02,0.08)$ & $0.54(0.25,0.18)$ \\
\hline \multicolumn{4}{|l|}{ Sex: } \\
\hline Male $(n=1222)$ & 1.00 & 1.00 & 1.00 \\
\hline Female $(n=970)$ & $1.64(1.16,2.17)$ & $1.20(0.79,1.48)$ & $1.28(1.04,1.58)$ \\
\hline \multicolumn{4}{|l|}{ Differentiation: } \\
\hline Well $(n=56)$ & 1.00 & 1.00 & 1.00 \\
\hline Moderate $(n=1187)$ & $0.84(0.38,1.87)$ & $1.15(0.60,2.19)$ & $1.26(0.67,2.38)$ \\
\hline Poor/undiff $(n=635)$ & $0.94(0.41,2.11)$ & $0.72(0.37,1.40)$ & $0.86(0.45,1.65)$ \\
\hline UK (n=314) & $0.77(0.33,1.81)$ & $0.54(0.27,1.09)$ & $0.67(0.34,1.321)$ \\
\hline \multicolumn{4}{|l|}{ Sub-site: } \\
\hline Proximal $(n=602)$ & 1.00 & 1.00 & 1.00 \\
\hline Transverse $(n=140)$ & $0.54(0.21,1.42)$ & $0.53(0.33,0.86)$ & $0.52(0.33,0.83)$ \\
\hline Distal $(n=631)$ & $1.25(0.80,1.97)$ & $0.87(0.66,1.15)$ & $0.88(0.67,1.15)$ \\
\hline Other/unspec. $(n=92)$ & $1.28(0.57,2.91)$ & $0.94(0.55,1.62)$ & $1.13(0.66,1.92)$ \\
\hline Rectosigmoid $(n=207)$ & $3.41(2.07,5.62)$ & $1.08(0.74,1.58)$ & $1.48(1.02,2.15)$ \\
\hline Rectum $(\mathrm{n}=520)$ & $7.43(4.99,11.06)$ & $1.01(0.75,1.35)$ & $1.64(1.23,2.19)$ \\
\hline \multicolumn{4}{|l|}{ Diagnostic period: } \\
\hline $1980-84(n=156)$ & 1.00 & 1.00 & 1.00 \\
\hline $1985-89(n=256)$ & $3.85(1.10,13.51)$ & $0.71(0.36,1.38)$ & $1.17(0.62,2.19)$ \\
\hline $1990-94(n=246)$ & $5.64(1.63,19.54)$ & $1.44(0.77,2.67)$ & $2.27(1.24,4.14)$ \\
\hline $1995-99(n=435)$ & $10.33(3.13,34.11)$ & $5.16(2.98,8.96)$ & $6.92(3.98,12.06)$ \\
\hline $2000-04(n=531)$ & $13.26(4.04,43.50)$ & $7.88(4.56,13.64)$ & $10.56(6.08,18.36)$ \\
\hline $2005-10(n=568)$ & $12.40(3.77,40.75)$ & $15.29(8.81,26.52)$ & $18.85(10.80,32.91)$ \\
\hline
\end{tabular}


Table 5. Relative Odds (95\% Confidence Limits) of Radiotherapy and/or Systemic Therapies for Colon Cancers Diagnosed with Distant Metastases: South Australian Major Public Hospitals, 1980-2010 Cases. [n=1465]*

\begin{tabular}{|c|c|c|c|c|}
\hline Groups & & Radiotherapy $(n=100)$ & Systemic $(n=512)$ & Either $(n=549)$ \\
\hline \multirow[t]{6}{*}{ Age at diagnosis (yrs.): } & $<40(\mathrm{n}=32)$ & 1.00 & 1.00 & 1.00 \\
\hline & $40-49(n=101)$ & $1.16(0.30,4.52)$ & $0.80(0.31,2.13)$ & $0.97(0.36,2.56)$ \\
\hline & $50-59(n=230)$ & $1.08(0.30,3.91)$ & $0.58(0.23,1.43)$ & $0.71(0.29,1.75)$ \\
\hline & $60-69(n=339)$ & $0.97(0.27,3.48)$ & $0.35(0.14,0.86)$ & $0.39(0.16,0.96)$ \\
\hline & $70-79(n=477)$ & $0.47(0.13,1.70)$ & $0.15(0.06,0.37)$ & $0.17(0.07,0.41)$ \\
\hline & $80+(n=286)$ & $0.24(0.06,1.01)$ & $0.03(0.01,0.09)$ & $0.04(0.02,0.11)$ \\
\hline \multirow[t]{2}{*}{ Sex: } & Male $(n=789)$ & 1.00 & 1.00 & 1.00 \\
\hline & Female $(n=676)$ & $1.59(1.02,2.48)$ & $1.12(0.91,1.52)$ & $1.19(0.92,1.54)$ \\
\hline \multirow[t]{4}{*}{ Differentiation: } & Well $(n=34)$ & 1.00 & 1.00 & 1.00 \\
\hline & Moderate $(n=768)$ & $0.60(0.19,1.84)$ & $0.82(0.36,1.87)$ & $0.94(0.41,2.15)$ \\
\hline & Poor/undiff $(n=448)$ & $0.81(0.26,2.53)$ & $0.47(0.20,1.08)$ & $0.61(0.27,1.42)$ \\
\hline & $\mathrm{UK}(\mathrm{n}=215)$ & $0.58(0.17,1.95)$ & $0.38(0.16,0.91)$ & $0.46(0.19,1.01)$ \\
\hline \multirow[t]{6}{*}{ Sub-site: } & Proximal $(n=602)$ & 1.00 & 1.00 & 1.00 \\
\hline & Transverse $(n=140)$ & $0.54(0.20,1.42)$ & $0.53(0.33,0.86)$ & $0.51(0.32,0.81)$ \\
\hline & Distal $(n=631)$ & $1.29(0.81,2.04)$ & $0.86(0.65,1.14)$ & $0.87(0.66,1.15)$ \\
\hline & Other/unspec. $(n=92)$ & $1.34(0.59,3.05)$ & $0.96(0.55,1.65)$ & $1.15(0.67,1.95)$ \\
\hline & Rectosigmoid & - & - & - \\
\hline & Rectum & - & - & - \\
\hline \multirow[t]{6}{*}{ Diagnostic period:** } & $1980-84(n=110)$ & 1.00 & 1.00 & 1.00 \\
\hline & $1985-89(n=166)$ & & $0.66(0.29,1.50)$ & $0.70(0.31,1.57)$ \\
\hline & $1990-94(n=165)$ & $3.69(1.24,10.92)$ & $1.45(0.69,3.06)$ & $2.02(0.98,4.17)$ \\
\hline & $1995-99(n=293)$ & $5.42(2.03,14.47)$ & $4.92(2.55,9.47)$ & $5.91(3.07,11.38)$ \\
\hline & $2000-04(n=341)$ & $5.75(2.16,15.29)$ & $6.79(3.53,13.06)$ & $7.68(3.99,14.78)$ \\
\hline & $2005-10(n=390)$ & $4.49(1.67,12.05)$ & $13.18(6.86,25.32)$ & $14.43(7.50,27.74)$ \\
\hline
\end{tabular}

*Adjusted for all variables in Table 5; **1980-1989 reference period used for radiotherapy due to low numbers

Table 6. Relative Odds (95\% Confidence Limits) of Radiotherapy and/or Systemic Therapies for Cancers of the Rectum/Sigmoid Junction and Rectum Diagnosed with Distant Metastases: South Australian Major Public Hospitals, 1980-2010 Cases. [n=727]* 3 multiple logistic regression analyses

\begin{tabular}{|c|c|c|c|c|}
\hline \multicolumn{2}{|l|}{ Groups } & \multirow{2}{*}{$\begin{array}{c}\text { Radiotherapy }(\mathbf{n = 2 1 4}) \\
1.00\end{array}$} & \multirow{2}{*}{$\frac{\text { Systemic }(\mathbf{n}=\mathbf{2 9 9})}{1.00}$} & \multirow{2}{*}{$\begin{array}{l}\text { Either }(\mathbf{n = 3 7 2}) \\
1.00\end{array}$} \\
\hline Age at diagnosis (yrs.): & $<40(\mathrm{n}=15)$ & & & \\
\hline & $40-49(n=55)$ & $1.11(0.31,4.04)$ & $0.92(0.20,4.14)$ & $1.97(0.43,8.99)$ \\
\hline & $50-59(n=123)$ & $1.12(0.33,3.77)$ & $0.59(0.14,2.45)$ & $1.03(0.25,4.25)$ \\
\hline & $60-69(n=230)$ & $0.94(0.28,18.6)$ & $0.44(0.11,1.79)$ & $0.62(0.16,2.47)$ \\
\hline & $70-79(n=200)$ & $1.79(0.24,3.09)$ & $0.20(0.05,0.81)$ & $0.37(0.09,1.46)$ \\
\hline & $80+(n=104)$ & $0.22(0.06,0.82)$ & $0.04(0.01,0.17)$ & $0.08(0.18,0.32)$ \\
\hline \multirow[t]{2}{*}{ Sex: } & Male $(n=433)$ & 1.00 & 1.00 & 1.00 \\
\hline & Female $(n=294)$ & $1.68(1.16,2.43)$ & $1.27(0.87,1.84)$ & $1.47(1.03,2.13)$ \\
\hline \multirow[t]{4}{*}{ Differentiation: } & Well $(n=22)$ & 1.00 & 1.00 & 1.00 \\
\hline & Moderate $(n=419)$ & $1.17(0.39,3.48)$ & $2.16(0.66,7.06)$ & $2.13(0.73,6.27)$ \\
\hline & Poor/undiff $(n=187)$ & $1.11(0.36,3.42)$ & $1.65(0.49,5.58)$ & $1.58(0.52,4.80)$ \\
\hline & UK (n=99) & $1.05(0.33,3.39)$ & $1.06(0.30,3.72)$ & $1.31(0.41,4.15)$ \\
\hline \multirow[t]{6}{*}{ Sub-site: } & Proximal & - & - & - \\
\hline & Transverse & - & - & - \\
\hline & Distal & - & - & - \\
\hline & Other/unspec. & - & - & - \\
\hline & Rectosigmoid $(\mathrm{n}=207)$ & $2.19(1.45,3.32)$ & $0.92(0.62,1.37)$ & $1.09(0.74,1.61)$ \\
\hline & Rectum $(\mathrm{n}=520)$ & 1.00 & 1.00 & 1.00 \\
\hline \multirow[t]{6}{*}{ Diagnostic period: } & $1980-84(n=46)$ & 1.00 & 1.00 & 1.00 \\
\hline & $1985-89(n=90)$ & $2.78(0.75,10.35)$ & $0.84(0.25,2.76)$ & $2.46(0.83,7.35)$ \\
\hline & $1990-94(n=81)$ & $2.85(0.75,10.80)$ & $1.59(0.51,4.92)$ & $3.12(1.05,9.30)$ \\
\hline & $1995-99(n=142)$ & $6.24(1.80,21.60)$ & $5.80(2.08,16.13)$ & $9.38(3.36,26.21)$ \\
\hline & $2000-04(n=190)$ & $9.30(2.72,31.79)$ & $10.67(3.87,29.44)$ & $19.83(7.10,55.36)$ \\
\hline & $2005-10(n=178)$ & $10.00(2.91,34.32)$ & $21.58(7.69,60.54)$ & $33.46(11.74,95.34)$ \\
\hline
\end{tabular}

*Adjusted for all variables in Table 6;**1980-1989 reference period used for radiotherapy due to low numbers

Corresponding increases were from $0 \%$ to $6.9 \%$ for colon and $6.5 \%$ to $39.0 \%$ for rectum (\&RS) cases. Percentages having radiotherapy were also higher for: $i$ ) Females than males: i.e., $17.9 \%$ compared with $9.8 \%(\mathrm{p}<0.001)$ ); ii) Cases under 80 years than $80+$ year olds: i.e., $16.5 \%$ compared with 4.4\% (MWp<0.001)); iii) Rectum (\&RS) compared with colon cases: i.e., $29.5 \%$ compared with $6.8 \%(\mathrm{p}<0.001)$.

A total of 811 colorectal cases $(37.0 \%)$ had systemic therapy, increasing from $11.5 \%$ for $1980-84$ to $57.1 \%$ for 
2005-10 (MWp<0.001). Corresponding increases were from $11.8 \%$ to $53.3 \%$ for colon and from $10.9 \%$ to $65.5 \%$ for rectum $(\& \mathrm{RS})$ cases $(\mathrm{MWp}<0.001)$. Percentages having systemic therapy were also higher for: $i$ ) Younger cases: e.g., $69.6 \%$ for those under 40 years, reducing with age to $9.5 \%$ for those aged $80+$ years $(\mathrm{MWp}<0.001)$; $i$ i) Sites other than transverse colon (note: the percentage having systemic therapy was $27.9 \%$ for transverse colon compared with $37.6 \%$ for other specified sub-sites collectively ( $\mathrm{p}=0.020)$ where differences did not apply.

Multivariable analyses: Multiple logistic regression analysis, with age, sex, differentiation, sub-site, and diagnostic period as predictors, indicated that variations in relative odds $(95 \% \mathrm{CLs})$ of radiotherapy/systemic therapies for colorectal cancer (Table 4), included: $i$ ) Reduced odds with increasing age to $0.47(0.23,0.99)$ for 60-69 years, $0.22(0.11,0.47)$ for $70-79$ years and 0.54 $(0.25,0.18)$ for $80+$ years, compared with those under 40 years. The reduction with increasing age was stronger for systemic therapies than radiotherapy such that the relative odds were $0.04(0.02,0.08)$ and $0.24(0.09,0.62)$ respectively for $80+$ years compared with under 40 years (Table 4); ii) Higher odds for females than males (RO = $1.28(1.04,1.58))$. This differences was more pronounced for radiotherapy $(\mathrm{RO}=1.64(1.16,2.17)$ than systemic therapies $(\mathrm{RO}=1.20(0.79,1.48))$; iii) Higher odds for rectum/sigmoid junction and rectum compared with the proximal colon reference category at $1.48(1.02,2.15)$ and $1.64(1.23,2.19)$ respectively, due to pronounced elevations for radiotherapy of $3.41(2.07,5.62)$ and 7.43 $(4.99,11.06)$ respectively. The lowest utilization was for cancers of the transverse colon $(\mathrm{RO}=0.52(0.33,0.83))$; $i v)$ Increasing relative odds for 1990-94 than 1980-84, increasing to $18.85(10.80,32.91)$ for $2005-10$, reflecting marked increases for both radiotherapy and systemic therapies.

Similar trends in relative odds applied for radiotherapy and systemic therapies for colon and rectum (\&RS) respectively, although results for radiotherapy for colon cancer by diagnostic period were difficult to interpret due to small numbers (Tables 5 \& 6).

Treatment of cases under 70 years of age: Percentages of cases diagnosed in 2005-10 receiving specified treatments were as follows: $i$ ) Surgery:- colorectal- $75.4 \%$; colon-78.1\%; rectum (\&RS)-71.2\%; ii) Radiotherapy or systemic therapy:- colorectal-78.9\%; colon-74.2\%; rectum (\&RS)-86.5\%; iii) Radiotherapy:- colorectal- 23.5\%; colon-9.6\%; rectum (\&RS)-46.0\%; iv) Systemic therapy:colorectal-75.1\%; colon-71.3\%; rectum (\&RS)-81.1\%

\section{Discussion}

Large increases in survival from colorectal cancer with distant metastases at diagnosis occurred between 1980-84 and $2005-10$, from $23 \%$ to $57 \%$ at one year, $10 \%$ to $35 \%$ at two years, and $3 \%$ to $16 \%$ at five years from diagnosis.

The five-year survival of $6 \%$ for $1980-89$ equated with the 6\% from U.S.A. SEER data for 1983-90 (Ries et al., 1994). By comparison, the five-year figure of $16 \%$ for 2005-10 was higher than the 13\% SEER figure for 2004-11 (Howlader et al., 2015). SACCR cases also had higher survivals than reported for the United Kingdom, with the five year survival of $10 \%$ for 2000-04 exceeding the 7-8\% for 2002-06 for the United Kingdom (Cancer Research UK, 2014).

A key finding was the approximate halving of the hazards ratio between $1980-84$ and $2005-10$ to 0.48 $(0.40,0.59)$, with similar reductions occurring for colon and rectum (\&RS) cancers, after adjusting for specified demographic and tumour characteristics.

It is expected that improved imaging could have led to stage shift and potentially an artificial contribution to improved stage-specific survivals. It is unlikely, however, that this would account for a halving of the hazards ratio. Table 1 data indicate that for changes in survival between 1980-1984 and 2005-2010 to have been artificial (i.e., for colorectal death outcomes to have remained unchanged), dates of diagnosis would need to have been brought forward on average by more than three years per case, which seems implausible.

It is also possible that increased diagnostic sensitivity could have led to distant metastases being detected that would not have been evident with previous diagnostic technology, leading to a less advanced mix of cases staged as metastatic. However, it seems implausible that this would have led to artificially recorded survival increases on the scale observed in this study (e.g., from $23 \%$ to $57 \%$ at one year and $10 \%$ to $35 \%$ at two years from diagnosis).

Real benefit is also suggested by the comparatively large reductions in hazards ratios from the late 1990s, which coincided with similar USA trends (Ries et al., 1994; Howlader et al., 2015), and also the addition of irinotecan and subsequently other systemic agents for treating metastatic disease (Saltz et al., 2001). The impact on outcomes of the later inclusion of biological agents as further treatment options is also supported by data from a more recently introduced specialized population-based metastatic colorectal cancer registry in South Australia (Charakidis et al., 2014). Collectively all data point to real and substantial survival gains, although the potential for an artificial contribution from stage shift is acknowledged.

The lower survivals from colorectal cancer in older cases (particularly those aged 80 years or more) are consistent with increased age-related frailty and comorbidity, and reduced use of systemic therapies and/ or radiotherapy to accommodate this. It is not possible to determine from the present data whether the best balance has been achieved in trade-offs between treatment compromises and patient capacity.

A more recent SA metastatic cancer registry initiative, including data on distant metastases occurring later in the course of the disease, suggested that for patients receiving systemic therapy, the outcomes were not dissimilar between those aged $80+$ years and those less than 80 years (Kumar et al., 2013). As a result, we may expect additional survival gains if it were possible to increase access to active therapy for this older population.

The higher survivals for rectum (\&RS) than proximal sub-sites (e.g., lower hazards ratios of $0.84(0.74,0.96)$ for rectum and $0.83(0.70,0.99)$ for rectum/sigmoid junction) are consistent with U.S.A. SEER data (Howlader et al., 2015) and not explained by differences in demographic 
or cancer characteristics or diagnostic period. A possible contributor is the more common use of systemic treatments, plus or minus radiotherapy, and the greater effectiveness of radiotherapy for local control, in addition to systemic therapies, for rectum ( $\& R S)$ sub-sites than proximal lesions (Schmoll et al., 2012; Price et al., 2015).

No associations were found of survivals with residential area measures, which is suggestive of a reasonable equity in service provision and outcomes at these hospitals. Reasons for the lower hazards ratio for females than males with rectum (\&RS) of 0.72 $(0.61,0.85)$ may reflect their greater use of radiotherapy $(\mathrm{RO}=1.68[1.16,2.43])$, although this is not certain.

Treatment of colorectal cancers diagnosed at a metastatic stage with systemic therapies, plus or minus radiotherapy, has increased significantly and this pattern is consistent with clinical guideline recommendations (USDHHS, 1990; NHMRC, 1999; ACN, 2005; Schmoll et al., 2012). This is considered to be a major contributor to observed survival gains. It is also possible that surgical treatment of discrete liver and pulmonary metastatic lesions is now making a contribution (Lubowski, 2015; Martenson et al., 2004; Dave et al., 2015). Other changes over the study period have included increased use of multidisciplinary teams in clinical management and greater access to medications through involvement in clinical trials and availability of pharmaceutical access programs, all of which could have contributed to better outcomes.

The percentage of colorectal cases diagnosed at less than 70 years of age, who had systemic therapy as part of primary treatment, increased to $75 \%$ in $1995-2010(81 \%$ for rectum (\&RS)). Based on the ratio of those treated to those offered this care indicated by national survey data (NCCI, 2000), it is likely that about $85 \%$ of these younger patients would have been offered systemic treatment. Meanwhile the percentage of corresponding metastatic rectal cases less than 70 years of age having radiotherapy as part of their primary treatment increased to $46 \%$ by 2005-10. Again, based on the ratio of those treated to those offered radiotherapy indicated by national survey data (NCCI, 2000), it would appear that about $63 \%$ of these younger patients would have been offered radiotherapy.

This study illustrates the value of clinical cancer registries in Australia for monitoring patterns of care and survival by stage (Neo et al., 2011; Due et al., 2012), including the specialized South Australian metastatic colorectal cancer registry (Padman et al., 2013). These registries provide important complementary information data to generic population-based registries (Coleman et al., 2011; Neo et al., 2011; Due et al., 2012; Padman et al., 2013; Beckmann et al., 2014b; Roder, 2014; Roder et al., 2014; Roder et al., 2015; Taheri et al., 2014; Roder \& Buckley, 2015). The electronic transfer of prognostic data from structured pathology reporting, and continuous updating of this reporting to account for new scientific evidence, will ensure that clinical registry data remain current for assessing health-system performance (Roder et al., 2014; Roder, 2014).

The value of clinical and population-based registries can be greatly increased when data extracts from these registries are linked, along with linkage to screening registries and other administrative datasets (Beckmann et al., 2014a; Roder et al., 2014; Roder, 2014).

In conclusions, $i$ ) There have been pronounced increases in survivals at major public hospitals in South Australia from colorectal cancers diagnosed at a metastatic stage; ii) Use of systemic therapies, radiotherapy and surgical intervention has increased substantially at these hospitals and changes in patterns of care are consistent with clinical guideline recommendations.

\section{Acknowledgements}

This study received funding support from the Royal Adelaide Hospital Health Services Charitable Gift Board, Cancer Council South Australia, the South Australia Health and Medical Research Institute, the University of South Australia, and the South Australian Department of Health. This funding support is gratefully acknowledged.

\section{References}

Australian Bureau of Statistics (ABS) (1998). 1996 census of population and housing. Socio-economic indexes for areas, ABS, Canberra.

Australian Institute of Health and Welfare (AIHW) (2012). Cancer survival and prevalence in Australia: period estimates from 1982 to 2010, Cancer Series No. 69. Cat. No. CAN 65, AIHW, Canberra.

Australian Institute of Health and Welfare (AIHW) (2013), Australian cancer incidence and mortality (ACIM) books, AIHW, Canberra. <available at: http://www.aihw.gov.au/ acim-books/>

Andre T, Boni C, Mounedji-Boudiaf L, et al (2004). Oxaplatin, fluorouracil, and leucovorin as adjuvant treatment for colon cancer. NEJM, 350, 2343-51.

Armitage P, Berry G (eds.) (1987). Statistical methods in medical research, Blackwell Scientific Publications, Oxford.

Australian Cancer Network Colorectal Cancer Guidelines Revision Committee (ACN) (2005). Guidelines for the prevention, early detection and management of colorectal cancer, Cancer Council Australia \& Australian Cancer Network, Sydney.

Australian Health Technology Advisory Committee (1997). Colorectal cancer screening, Commonwealth Department of Health and Family Services, Canberra.

Beckmann KR, Bennett A, Young GP, et al (2014a). Treatment patterns among colorectal cancer patients in South Australia: a demonstration of the utility of population-based data linkage. J Eval Clin Pract, 20, 467-77.

Beckmann KR, Roder DM, Hiller JE, et al (2014b). Influence of mammographic screening on breast cancer incidence trends in South Australia. Asian Pac J Cancer Prev, 15, 3105-12.

Bonett A, Roder D, Esterman A (1988). Cancer case-survival rates for South Australia: a comparison with US rates and a preliminary investigation of time trends. Med J Aust, 148, 556-9.

Cancer Research UK (2014). Bowel cancer survival statistics. London: Cancer Research UK.

Charakidis M, Price TJ, Beeke C, et al (2014). Survival improvements associated with access to biologic agents: Results from the South Australian (SA) metastatic colorectal (mCRC) Registry. J Clin Oncol, 32, suppl; abstr e17616.

Cole SR, Tucker GR, Osborne JM, et al (2013). Shift to earlier stage at diagnosis as a consequence of the National Bowel 
Cancer Screening Program. Med J Aust, 198, 327-30.

Coleman MP, Forman D, Bryant H, et al (2011). Cancer survival in Australia, Canada, Denmark, Norway, Sweden, and the UK, 1995-2007 (the International Cancer Benchmarking Partnership): an analysis of population-based cancer registry data. Lancet, 377, 127-38.

Dave RV, Pathak S, White AD, et al (2015). Outcome after liver resection in patients presenting with simultaneous hepatopulmonary colorectal metastases. Br J Surg, 102, 261-8.

Department of Health \& (AIHW) Australian Institute of Health and Welfare (2008). National Bowel Screening Program Monitoring Report, Canberra, AIHW, Cancer Series 44. Cat. no. 40

Due SL, Wattchow DA, Sweeney JL, et al (2012). Colorectal cancer surgery 2000-2008: evaluation of a prospective database. ANZ J Surg, 82, 412-9.

Ferlay J, Soerjomataram I, Ervik M, et al (2013). Cancer incidence and mortality worldwide: IARC CancerBase No.11. Lyon, France: IARC <available at: http://globocan. iarc.fr>.

Hardcastle JD, Chamberlain JO, Robinson MH, et al (1996). Randomised controlled trial of faecal-occult-blood screening for colorectal cancer. Lancet, 348, 1472-7.

Hewitson P, Glasziou P, Watson E, et al (2008). Cochrane systematic review of colorectal cancer screening using the fecal occult blood test (hemoccult): an update. $A m J$ Gastroenterol, 103, 1541-9.

Howlader N, Noone AM, Krapcho M, et al (eds.) (2015), SEER Cancer Statistics Review, 1975-2012, National Cancer Institute, Bethesda, MD.

Jong KE, Smith DP, Yu XQ, et al (2004). Remoteness of residence and survival from cancer in New South Wales. Med J Aust, 180, 618-22.

Jorgensen OD, Kronborg O, Fenger C (2002). A randomised study of screening for colorectal cancer using faecal occult blood testing: results after 13 years and seven biennial screening rounds. Gut, 50, 29-32.

Kronborg O, Fenger C, Olsen J, et al (1996). Randomised study of screening for colorectal cancer with faecal-occult-blood test. Lancet, 348, 1467-71.

Kronborg O, Fenger C, Sondergaard O, et al (1987). Initial mass screening for colorectal cancer with fecal occult blood test. A prospective randomized study at Funen in Denmark. Scand J Gastroenterol, 22, 677-86.

Kumar R, Jain K, Beeke C, et al (2013). A population-based study of metastatic colorectal cancer in individuals aged $>=$ 80 years: findings from the South Australian clinical registry for metastatic colorectal cancer. Cancer, 119, 722-8.

Lubowski DZ (2015). Rectal cancer: the evolving role of adjuvant radiotherapy. ANZ J Surg, 85, 99-100.

Mandel JS, Church TR, Bond JH, et al (2000). The effect of fecal occult-blood screening on the incidence of colorectal cancer. $N$ Engl J Med, 343, 1603-7.

Martenson JA Jr, Willett CG, Sargent DJ, et al (2004). Phase III study of adjuvant chemotherapy and radiation therapy compared with chemotherapy alone in the surgical adjuvant treatment of colon cancer: results of intergroup protocol 0130. J Clin Oncol, 22, 3277-83.

National Cancer Control Initiative (NCCI) (2000). The national colorectal cancer care survey. Australian clinical practice in 2000, Clinical Governance Unit, Melbourne.

Neo EL, Beeke C, Price T, et al (2011). South Australian clinical registry for metastatic colorectal cancer. ANZ J Surg, 81, 352-7.

(NHMRC) National Health and Medical Research Council (1999), Clinical Practice Guidelines. Prevention, early detection and management of colorectal cancer, Commonwealth of Australia, Canberra.

Padman S, Padbury R, Beeke C, et al (2013). Liver only metastatic disease in patients with metastatic colorectal cancer: impact of surgery and chemotherapy. Acta Oncol, 52, 1699-706.

Price TJ, Beeke C, Ullah S, et al (2015). Does the primary site of colorectal cancer impact outcomes for patients with metastatic disease? Cancer, 121, 830-5.

Price TJ, Segelov E, Burge M, et al (2014). Current opinion on optimal systemic treatment for metastatic colorectal cancer: outcome of the ACTG/AGITG expert meeting ECCO 2013. Expert Rev Anticancer Ther, 14, 1477-93.

Ries LAG, Miller BA, Hankey BF, et al (1994). SEER cancer statistics review, 1973-1991, National Cancer Institute, Bethesda, MD.

Roder D, Karapetis CS, Wattchow D, et al (2015). Colorectal cancer treatment and survival: the experience of major public hospitals in South Australia over three decades. Asian Pac J Cancer Prev, 16, 2431-40.

Roder D (2014). What roles should population-based cancer registries be playing in the 21 st century? Reflections on the Asian Cancer Registry Forum, Bangkok, February 2014. Asian Pac J Cancer Prev, 15, 1895-6.

Roder D, Buckley E (2015). Translation from clinical trials to routine practice: How to demonstrate community benefit. Asian Pac J Clin Oncol, [Epub ahead of print].

Roder DM, Fong KM, Brown MP, et al (2014). Realising opportunities for evidence-based cancer service delivery and research: linking cancer registry and administrative data in Australia. Eur J Cancer Care (Engl), 23, 721-7.

(SACR) South Australian Cancer Registry (1999). Epidemiology of cancer in South Australia. incidence, mortality, and survival 1977 to 1998 . Incidence and mortality 1998, analysed by type and geographical location, Epidemiology Branch, Department of Health, Adelaide.

(SACR) South Australian Cancer Registry (2007). Cancer in South Australia 2004, with incidence projections to 2007, Epidemiology Branch, Department of Health, Adelaide.

Saltz LB, Douillard JY, Pirotta N, et al (2001). Irinotecan plus fluorouracil/leucovorin for metastatic colorectal cancer: a new survival standard. Oncologist, 6, 81-91.

Schmoll HJ, Van Cutsem E, Stein A, et al (2012). ESMO Consensus Guidelines for management of patients with colon and rectal cancer. A personalized approach to clinical decision making. Ann Oncol, 23, 2479-516.

Scholefield JH, Moss S, Sufi F, et al (2002). Effect of faecal occult blood screening on mortality from colorectal cancer: results from a randomised controlled trial. Gut, 50, 840-4.

STATA (2012), Data analysis and statistical software, Version 12, StataCorp LP, College Station, Texas.

Taheri N, Fazel A, Mahmoodzadeh H, et al (2014). Epidemiology of female reproductive cancers in Iran: results of the Gholestan Population-based Cancer Registry. Asian Pac J Cancer Prev, 15, 8779-82.

U.S. Department of Health and Human Services (USDHHS). National Institutes of Health (NIH) (1990). NIH consensus development program: adjuvant therapy for patients with colon and rectum cancer, NIH consensus statement online. April 16-18 1990, 1-25. <available at: http://consensus. nih.gov/1990/1990adjuvanttherapycolonrectalcancer079h tml.htm> 